\title{
The Anxiety Depression Pathway Among Men Following a Prostate Cancer Diagnosis: Cross-Sectional Interactions Between Anger Responses and Loneliness
}

American Journal of Men's Health May-June I-9 (C) The Author(s) 2021 Article reuse guidelines: sagepub.com/journals-permissions DOI: $10.1177 / \mid 5579883211023699$ journals.sagepub.com/home/jmh (S)AGE

\author{
Simon M. Rice ${ }^{1,2}$, David Kealy ${ }^{3}$, John S. Ogrodniczuk ${ }^{3}$, \\ Zac E. Seidler ${ }^{1,2}(\mathbb{D})$, Gabriela Montaner ${ }^{4}$, \\ Suzanne Chambers ${ }^{5,6}$, and John L. Oliffe ${ }^{4,7}$ (iD)
}

\begin{abstract}
Anger has been a largely neglected emotion in prostate cancer research and intervention. This paper highlights the role of anger in the anxiety depression pathway among men with prostate cancer, and whether its impact is dependent on loneliness. Data are presented from a sample of men with prostate cancer $(N=105, M=69.12$ years, prostatectomy $=63.8 \%$ ) and analysed using conditional process analysis. Dimensions of anger were evaluated as parallel mediators in bi-directional anxiety and depression pathways. Loneliness was evaluated as a conditional moderator of identified significant mediation relationships. Moderate severity depression (16.5\%) was endorsed more frequently than moderate severity anxiety $(8.6 \%, p=.008)$, with $19.1 \%$ of the sample reporting past two-week suicide ideation. Consistent with hypotheses, anger-related social interference (but not other dimensions of anger) significantly mediated the anxiety-depression pathway, but not the reverse depression-anxiety pathway. This indirect effect was conditional on men experiencing loneliness. Sensitivity analyses indicated the observed moderated mediation effect occurred for affective, but not somatic symptoms of depression. Findings support anger-related social interference (as opposed to anger frequency, intensity, duration or antagonism) as key to explaining the previously established anxietydepression pathway. Results underscore the need for enhanced psychosocial supports for men with prostate cancer, with a particular focus on relational aspects. Supporting men with prostate cancer to adaptively process and manage their anger in ways that ameliorate negative social consequences will likely enhance their perceived social support quality, which may in turn better facilitate post-diagnosis recovery and emotional adjustment.
\end{abstract}

\section{Keywords \\ prostate cancer, anger, depression, anxiety, help-seeking, social support}

Received April 16, 2021; accepted May 17, 2021

Research supports the existence of a mixed anxietydepression symptom profile in men with prostate cancer (Sharpley et al., 2007; Sharpley \& Christie, 2007; Sharpley et al., 2010), suggesting substantial symptomatic overlap in these syndromes. This finding is consistent with meta-analytic data in the general population $(N=$ $88,336)$ that suggests a bidirectional temporal relationship between anxiety and depression, with a marginally stronger longitudinal effect of anxiety leading to depression $(r=.34)$, compared to depression leading to anxiety $(r=.31)$ (Jacobson \& Newman, 2017). More specific to depression, researchers have identified two distinct symptom clusters experienced by men within the major depressive disorder diagnostic criteria pertaining to affective (e.g., anhedonia, suicide ideation) and somatic (e.g., fatigue, sleep disturbance) symptoms (Rice et al., 2019). The relevance of this affective and somatic depression symptom distinction has not been thoroughly explored in men with prostate cancer.

Social support is widely acknowledged as a protective factor in ameliorating the psychological burden associated with prostate cancer diagnosis (Oliffe et al., 2009). 
Identifying and addressing factors that impede social support among men with prostate cancer is therefore essential. While it is known that depression and anxiety can be precipitated by social difficulties in middle- and olderaged males (George et al., 1989), loneliness has also been implicated in the association between social network integration and depression (Santini et al., 2016), and anxiety (Domènech-Abella et al., 2019). Loneliness is an established risk factor for depression and anxiety (and suicide attempt) in the general population (Beutel et al., 2017), but there is a lack of research into the impact of loneliness in men with prostate cancer (Ervik et al., 2010). Potentially, men experiencing loneliness can be at elevated risk of experiencing difficulties in the quality and depth of their social relationships by virtue of having fewer meaningful social contacts, and a smaller social network to draw on. This may be a particularly important factor in the presence of men with prostate cancer experiencing symptoms of anxiety and depression.

While symptoms of anxiety, depression and loneliness have been examined in populations of men with prostate cancer (Chambers et al., 2018; Sharp et al., 2016), the interpersonal consequences of anger (i.e., anger-related social interference; where anger inhibits relationship quality) are less well documented (Gerhart et al., 2017; King et al., 2015). Given the established link between anger and psychological distress in the general male population (Oliffe et al., 2019), and prospective evidence suggesting a weak association between anger control and prostate cancer risk (White et al., 2007), the construct of anger warrants further exploration in the experience of men living with prostate cancer.

Normative gender socialisation processes sanction men's experience and expression of anger at times of psychological distress, in contrast to internalising symptoms of depression and anxiety - which may confer a perception of weakness, vulnerability, and self-stigma (Rice et al., 2013). Notwithstanding this, research shows that chronic experiences of anger and associated emotions and behaviours (e.g., irritability, aggression, frustration) can negatively influence immune functioning and thus general well-being (Brod et al., 2014), which, by extension, can impede men's interpersonal relationships (Overall et al., 2016). Underscoring this, in men treated for prostate cancer, maladaptive suppression of anger has been found to mediate the relationship between optimism and natural killer cell cytotoxicity, a finding that highlights the immunological benefit of men's adaptive processing and, where appropriate, expression of anger (Penedo et al., 2006).

Better understanding anger and its consequences will serve an important aspect of prostate cancer management and recovery, potentially informing future interventions for men as these factors can influence oncology outcomes (Galon et al., 2012). Men prone to anger are more likely to hold pessimistic and discrepant attitudes in relation to 5 -year prostate cancer prognosis relative to their treating physician (Gerhart et al., 2017). Additionally, anger is commonly reported by men with prostate cancer and may be an important diagnostic marker of psychosocial challenges for this population (Rice et al., 2018). A Finnish population-based study $(N=1,239)$ found that irritability $(46 \%)$ was the second most common psychological symptom reported (after worry, 57\%) five years post prostate cancer diagnosis (Lehto et al., 2017), and qualitative and cross-sectional research supports the importance of anger in men's experiences post-diagnosis (Carter et al., 2011; Fitch et al., 2000).

In cases where men's anger specifically impacts the quality and depth of their social relationships (e.g., angerrelated social interference), men with prostate cancer may be more likely to experience symptoms of common mental health disorders such as anxiety and depressionboth disorders have elevated prevalence in men with prostate cancer relative to same age men in the general population (Watts et al., 2014). From a familial relationship perspective, research has shown that psychological distress in men with prostate cancer (and their partners) was in the normal range when positive perceived support from family was reported, whereas psychological distress was high when family support was perceived as absent or low (Baider et al., 2003). Given the interpersonal aspect of common mental disorders like anxiety and depression-both have a strong relational component in their respective diagnostic criteria (American Psychiatric

\footnotetext{
'Orygen, Parkville, Melbourne, VIC, Australia

${ }^{2}$ Centre for Youth Mental Health, The University of Melbourne, Australia

${ }^{3}$ Department of Psychiatry, University of British Columbia, Vancouver, BC, Canada

${ }^{4}$ School of Nursing, University of British Columbia, Vancouver, BC, Canada

${ }^{5}$ Faculty of Health Sciences, Australian Catholic University, Brisbane, Australia

${ }^{6}$ Faculty of Health, University of Technology Sydney, Sydney NSW, Australia

${ }^{7}$ Department of Nursing, The University of Melbourne, Australia
}

\section{Corresponding Author:}

Simon M. Rice, Orygen, Centre for Youth Mental Health, The University of Melbourne, 35 Poplar Rd, Parkville, VIC 3052, Australia.

Email: simon.rice@orygen.org.au 
Association, 2013) - anger-related social interference may be comparatively more salient than general domains of anger dimensions such as frequency, severity, duration, or antagonism. Moreover, anger-related social interference may be amplified by men's experience of loneliness. Research has yet to explore this.

The current study sought to test the above associations using a conditional process analysis approach, testing dimensions of anger as parallel mediators in the anxiety depression pathway, and exploring whether indirect effects of anger were conditional on loneliness. It was expected that anger-related social interference would report stronger bivariate associations with anxiety and depression than other related indices of anger, and that associations for anger-related social interference would be stronger for the affective depression domain relative to the somatic domain. It was also hypothesised that loneliness would moderate pathways between anxiety and anger domains, and anger domains and depression (e.g., moderate mediation). The reverse depression-anxiety model was expected to yield a non-significant effect, supporting the putative directionality of this hypothesised pathway.

\section{Method}

\section{Participants and Design}

Data are reported from a cross-sectional convenience sample of 105 Canadian men. Inclusion criteria were selfidentification as having prostate cancer, ability to read and comprehend English and internet access. Reporting conforms to the STROBE statement for observational studies (Von Elm et al., 2007).

\section{Measures}

Anger. The Dimensions of Anger Responses (DAR-5; (Novaco et al., 2012) is a five-item scale used to assess anger frequency, intensity, duration, interpersonal aggressiveness, and the extent to which anger interferes with interpersonal relationships (Forbes et al., 2014). Each item is presented on a 5 -item Likert scale ranging from not at all (0) to very much (4). The DAR-5 is a reliable and valid screening measure of common anger reactions (Forbes et al., 2014).

Anxiety. The General Anxiety Disorder Scale (GAD-7; (Spitzer et al., 2006) is a seven-item scale used to measure respondent's anxiety and was developed from the DSM-IV-TR (Frances et al., 2000) diagnostic criteria for GAD (unchanged for the DSM-5, (American Psychiatric Association, 2013). Respondents are asked to answer items (e.g., "Feeling nervous, anxious, or on edge") by endorsing one of four options: not at all (0), several days
(1), more than half of the days (2), and nearly every day (3) for how they have felt "during the last two weeks." Possible scores range from 0 to 21 . Scores of $0-4$ indicate "minimal" anxiety; 5-9 indicate "mild" anxiety; 10$14=$ "moderate" anxiety, and GAD-7 scores greater than 14 indicate "severe" anxiety (Spitzer et al., 2006).

Depression. Patient Health Questionnaire - Depression Module (PHQ-9; (Kroenke et al., 2001) is a nine-item screening instrument used to determine depression severity of respondents. For each item (e.g., "Little interest or pleasure in doing things") respondents are asked to assess how much each symptom bothered them in the last 14-day period using the responses: not at all (0), several days (1), more than half of the days (2), and nearly every day (3). The sum score (range 0-27) indicates the degree of depression, with scores of $\geq 5, \geq 10$, and $\geq 15$ representing mild, moderate, and severe levels of depression, respectively. The validity of the PHQ-9 has been demonstrated and it has been shown to perform well in assessing depression in cancer patients (Hinz et al., 2016). Previously, using a confirmatory factor analytic approach, the validity of the PHQ-9 affective (depressed mood, anhedonia, guilt, suicidal ideation) and somatic domains (concentration, appetite, psychomotor symptoms, sleep, fatigue) has been estabsoihed in men (Rice et al., 2019).

Loneliness. An abbreviated version of the UCLA Loneliness Scale (UCLA Loneliness Scale-3; (Russell, 1996) was used to assess feelings of loneliness or social isolation. The scale has three items including: "How often do you feel you lack companionship," "how often do you feel left out," and "how often do you feel isolated." The response categories are hardly ever (1), some of the time (2), and often (3). Higher scores indicate greater loneliness. The UCLA Loneliness Scale has shown excellent reliability and validity across studies, including studies with cancer patients (Jaremka et al., 2014). A score of $\geq 2$ reflects social isolation and $\geq 6$ reflects loneliness (Steptoe et al., 2013).

\section{Procedure}

Ethics review was approved by the University of British Columbia (H12-00573). The survey was launched in June, 2019, focussing on the issue of mental health symptoms in men with prostate cancer. Participation provided an opportunity for respondents to be entered into a $\$ 500$ cash prize draw by completing the survey. Recruitment occurred via social media channels and the survey was embedded in an online prostate cancer psychosocial resource (www.ifiweretom.ca), available for 4 months through October, 2019. The survey landing page provided details about the study including informed consent, confidentiality regarding respondents' demographic data 
and its separate password-protected storage from their survey responses. On completion of the survey, respondents were provided a URL link to the men's depression website www.headsupguys.org.

\section{Data Analysis}

Descriptive statistics and non-parametric tests were used to characterise the sample and Pearson correlation coefficients examined bivariate associations. Two-tailed Fisher $r$ to $z$ transformations explored correlation differences between anger social interference and other domains of anger with anxiety and depression (Eid et al., 2017). A Wilcoxon signed-rank test evaluated any difference in the proportion of men endorsing moderate severity depression (PHQ-9 $\geq 10$ ) versus anxiety (GAD-7 $\geq 10$ ). Conditional process models were evaluated in SPSS 26.0 using the PROCESS macro (Hayes, 2017), with the five DAR-5 dimensions evaluated as parallel mediators between anxiety (IV) and depression (DV), including covariates: years since prostate cancer diagnosis, prostatectomy and androgen deprivation therapy. Models evaluated loneliness as a moderator of the anxiety to anger pathway (PROCESS model 7) as well as simultaneously moderating the anger to depression pathway (PROCESS model 58). Finally, separate sensitivity analyses were undertaken using PROCESS to examine whether any observed conditional process effects occurred separately when affective depression symptoms versus somatic depression symptoms were treated as dependent variables in the model. In each case, 10,000 bootstrap resamples with $95 \%$ CIs were utilised. The bootstrapped approach to testing moderated mediation has the advantage not requiring assumptions of normality of the sampling distribution.

\section{Results}

Sample mean age was 69.12 years $(S D=8.67)$, with $96.2 \%(n=101)$ of participants identifying as heterosexual. Mean years since prostate cancer diagnosis was 7.4 $(S D=6.70)$. Current treatment was reported by $29.4 \%(n$ $=30)$, prostatectomy reported by $63.8 \%(n=67)$, androgen deprivation therapy for $20 \%(n=21)$. Outcome data were complete with the exception of two cases, each missing a single item on the PHQ-9, which were subject to listwise deletion. Outcome measures demonstrated satisfactory reliability (GAD-7 $\alpha=.90$; PHQ-9 $\alpha=.88$; UCLA; $\alpha=.87)$, as did the PHQ-9 affective domain ( $\alpha=$ $.86)$ and somatic domain $(\alpha=.73)$. On average, the sample were in the normal range for symptoms of anxiety $(M$ $=3.24, S D=3.75)$ and depression $(M=4.95, S D=$ 4.74). Based on the PHQ-9 and GAD-7 cut-offs, a Wilcoxon signed-rank test showed that moderate severity
Table I. Clinical Cut-offs for PHQ-9 and GAD-7.

\begin{tabular}{lccc}
\hline & PHQ-9 & & GAD-7 \\
\cline { 2 - 2 } Scale cut-off (score range) & $\%(n)$ & & $\%(n)$ \\
\hline Normal $(0-4)$ & $66(68)$ & & $77.3(77)$ \\
Mild (5-9) & $15.5(16)$ & & $16.2(17)$ \\
Moderate (10-14) & $13.6(14)$ & & $9.5(10)$ \\
Moderate-severe $(\geq 15)$ & $4.9(5)$ & & $1.0(1)$ \\
\hline
\end{tabular}

depression $(16.5 \%)$ was endorsed more frequently than moderate severity anxiety $(8.6 \% ; Z=-2.83, p=.008$; see Table 1). One fifth of participants $(19.1 \% ; n=20)$ reported past two-week suicide or self-harm ideation. Mean loneliness scores were relatively low $(M=1.16, S D$ $=1.58)$, although $25.7 \%(n=27)$ scored at or above the upper quartile of the total score $(\geq 6)$, with $59.0 \%(n=$ 62) scoring above threshold $(\geq 2)$ for social isolation. There was no association between time since diagnosis and scores on the PHQ-9 $(r=-.16, p=.10)$ or GAD-7 $(r=-.15, p=.12)$, nor were there mean depression or anxiety differences for men on current treatment (PHQ-9 $p=.077$; GAD-7 $p=.257$ ), or ADT intervention specifically (PHQ-9 $p=.834$; GAD-7 $p=.344$ ).

Almost all study variables reported robust moderatestrong associations (see Table 2). Of note, past 2-week suicide and self-harm ideation demonstrated equivalent magnitude correlations with both anxiety and depression. Results provided partial support for the hypothesis that anger social interference would have stronger correlations with anxiety and depression relative to other anger domains. For anxiety, this was the case for social interference relative to antagonism ( $p=.016)$ but not anger frequency, intensity or duration ( $p$ 's $>.05)$. For depression, stronger associations were observed for social interference relative to antagonism $(p<.001)$, and anger intensity and duration ( $p$ 's $=.024)$, but not anger frequency $(p$ $=.08$ ). Contrary to the hypothesis that affective depression symptoms would show a stronger correlation with anger-related social interference relative to somatic depression symptoms, no difference was observed in the correlation magnitude $(p=.256)$.

In partial support of the hypothesis, conditional process analysis indicated that loneliness moderated the mediation effect of anger-related social interference on the relationship between anxiety and depression (omnibus moderated mediation effect index $=.045,95 \%$ CI $.01-.10$; see Supplementary Table 1 for coefficients). Loneliness did not moderate the direct effect of anxiety predicting depression, and contrary to prediction, moderated mediation was not observed for the anger to depression pathway, or when tested as a simultaneous moderator. For the significant moderated mediation model, anger domains of intensity, frequency, duration, and antagonism toward others were 
Table 2. Bivariate Associations Between Study Variables.

\begin{tabular}{|c|c|c|c|c|c|c|c|c|c|c|c|}
\hline & $\mathrm{I}$. & 2. & 3. & 4. & 5. & 6. & 7. & 8. & 9. & 10. & II. \\
\hline I. GAD-7 Total & - & & & & & & & & & & \\
\hline 2. PHQ-9 Total & $.85 * * *$ & - & & & & & & & & & \\
\hline 3. PHQ-9 Affective & $.83 * * *$ & $.93 * * *$ & - & & & & & & & & \\
\hline 4. PHQ-9 Somatic & $.77 * * *$ & $.94 * * *$ & $.77 * * *$ & - & & & & & & & \\
\hline 5. DAR-5 Total & $.64 * * *$ & $.61^{* * *}$ & $.59 * * *$ & $.55 * * *$ & - & & & & & & \\
\hline 6. UCLA Loneliness & $.47 * * *$ & $.54 * * *$ & $.54 * * *$ & $.48 * * *$ & $.43 * * *$ & - & & & & & \\
\hline 7. DAR-5 Frequency & $.55 * * *$ & $.54 * * *$ & $.49 * * *$ & $.52 * * *$ & $.82 * * *$ & $.30 * *$ & - & & & & \\
\hline 8. DAR-5 Intensity & $.54 * * *$ & $.46 * * *$ & $.44 * * *$ & $.42 * * *$ & $.86 * * *$ & $.24^{*}$ & $.65 * * *$ & - & & & \\
\hline 9. DAR-5 Duration & $.5 I^{* * *}$ & $.46 * * *$ & $.47 * * *$ & $.40 * * *$ & $.89 * * *$ & $.38 * * *$ & $.56 * * *$ & $.76 * * *$ & - & & \\
\hline 10. DAR-5 Antagonism & $.35 * * *$ & $.25^{*}$ & $27 * *$ & $.21^{*}$ & $.69 * * *$ & .15 & $.40 * * *$ & $.52 * * *$ & $.69 * * *$ & - & \\
\hline II. DAR-5 Social & $.57 * * *$ & $.64 * * *$ & $.64 * * *$ & $.57 * * *$ & $.75^{* * *}$ & $.61 * * *$ & $.53 * * *$ & $.46 * * *$ & $.60 * * *$ & $.40 * * *$ & - \\
\hline 12. Suicide ideation & $.74 * * *$ & $.75 * * *$ & $.80 * * *$ & $.62 * * *$ & $.56 * * *$ & $.37 * * *$ & $.48 * * *$ & $.49 * * *$ & $.43 * * *$ & $.33 * *$ & $.49 * * *$ \\
\hline
\end{tabular}

Note. Italicised items refer to single item measures where 4-8 = DAR-5 items; $9=$ PHQ-9 item $9 ; *^{* *} p<.00 \mathrm{I},{ }^{*} p<.0 \mathrm{I},{ }^{*} p<.05$

not significant mediators, though anger-related social interference was. Bootstrapped probing indicated that the conditional (i.e., moderated) mediation effect occurred for those men with a loneliness score on the UCLA Loneliness Scale of $\geq 6$ (effect $=.16, \mathrm{SE}=.08,95 \%$ CI .02-.34, $p<$ $.001)$, representing the uppermost quartile $(25.7 \%)$ of the loneliness distribution. The full model was significant, and accounted for almost $80 \%$ of the variance in depression $\left(F(9,92)=37.87, M S E=5.25, R^{2}=0.78, p<\right.$ .001 ), with the anxiety $\times$ loneliness (moderation) interaction for anger-related social interference accounting for an additional $5 \%$ of variance $\left(F(1,96)=11.18, \Delta R^{2}=\right.$ $0.0544, p=.001)$. Neither of the three covariates were significant in the model. Coefficients are presented in Figure 1. In contrast to these effects, the reverse pathway of depression predicting anxiety failed to yield significant indirect or conditional effects. Sensitivity (conditional process) analyses found that the moderated mediation effect was observed for affective symptoms of depression (moderated mediation effect index $=.022,95 \%$ CI .01.05 ), but not for somatic symptoms of depression given the $95 \%$ CI straddled zero (moderated mediation effect index $=.022,95 \%$ CI .01- -.01). Supporting data are presented in Supplementary Tables 2 and 3.

\section{Discussion}

The present findings highlight associations between anxiety, anger-interference, loneliness, and depression for men who have received a prostate cancer diagnosis. While prostate cancer has a 5 -year survival rate of $93 \%$ (Canadian Cancer Society, 2021), many men experience prostate cancer as a lifelong illness, in part, because primary treatments can reduce quality of life (Chambers et al., 2018; Dunn et al., 2020; Resnick \& Penson, 2012). That the present sample were on average 7.4 years post-initial diagnosis, highlights the relevance across the illness trajectory for men with prostate cancer. In the present sample, symptoms of depression and anxiety of at least mild severity and suicide/self-harm ideation were frequently observed (in $34 \%, 22.7 \%$, and $19.1 \%$ of participants respectively. These rates are higher than those observed in a recent meta-analysis of men with prostate cancer $(17.1 \%, \quad 16.9 \%$, and $9.5 \%$ respectively; Brunckhorst et al., 2020), which in turn, were also higher than the general community (Hinz et al., 2016; Vasiliadis et al., 2015). Similarly, a higher rate (25.7\%) scored above the loneliness threshold on the UCLA Loneliness Scale relative to similar-aged community sample (18.9\%; 65.6 years; [Tymoszuk et al., 2019]), with the majority of respondents (59\%) scoring above the threshold for social isolation. Hence, while the majority of participants were in the non-clinical range for depression and anxiety, this sample nonetheless reported affective and loneliness symptoms above expected community rates. This suggests that having prostate cancer may influence levels of distress and social connectedness for this population, which aligns with previous work highlighting the burden to quality of life for these men (Brunckhorst et al., 2020).

Contrary to our hypothesis, loneliness did not moderate all pathways in the anxiety-depression mediation model; however a significant conditional process effect was observed for the predictive relationship between anxiety symptoms and anger-related social interference. As this effect occurred for those men in the uppermost $25 \%$ of the loneliness distribution (in this instance, $\geq 6$ on the UCLA brief scale), results show that for these lonely men, relational difficulties as a consequence of anger account for a part of the predictive association between anxiety and depression. In practical terms, this effect indicates that loneliness and anxiety interact, conferring a heightened risk of depression through relational aspects 


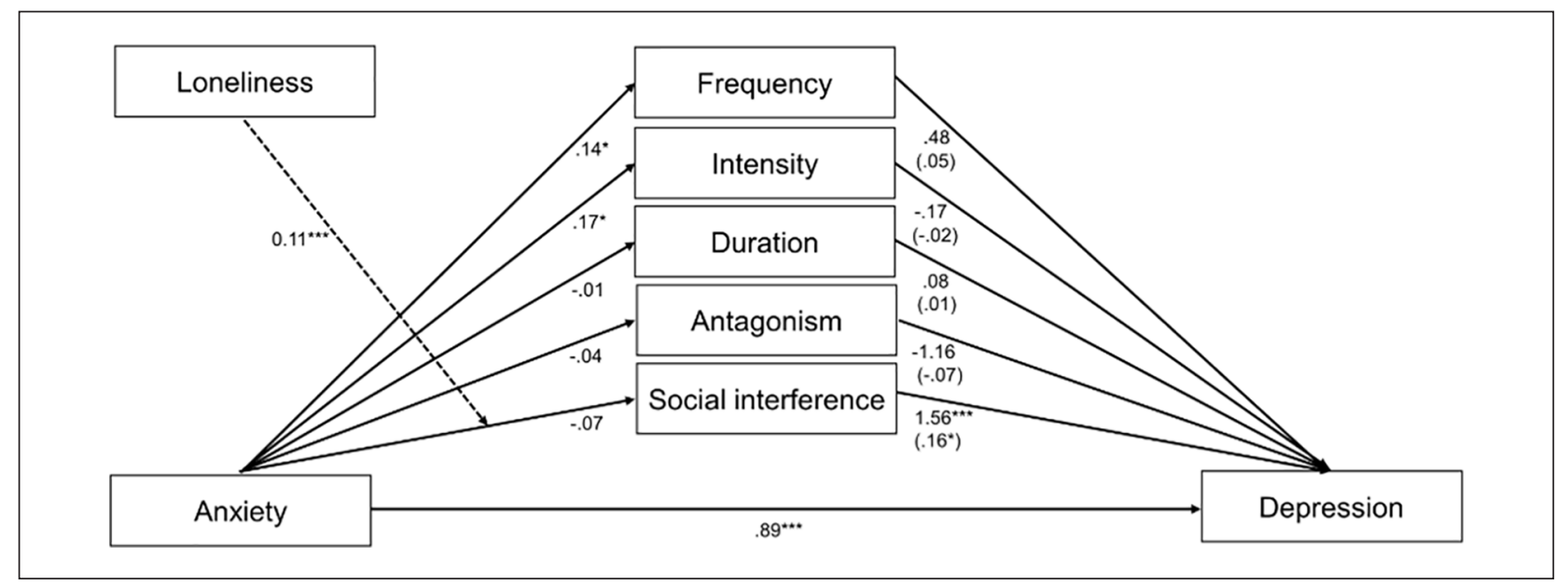

Figure I. Moderated mediated model.

of anger. These results are important because anxiety, loneliness and regulation of the social effects of anger are all readily modifiable factors, and clinical attention (e.g., assessment and intervention) to these domains may have a demonstrable upstream impact on reducing depression in men with prostate cancer.

An important caveat of the observed moderated mediation effect was the salience for affective but not somatic symptoms of depression. At a phenomenological level, this highlights the contribution of relational factors toward the emotional experience of men with prostate cancer. While this population have physical health needs - including somatic depression symptoms - it was the affective symptoms of depression (e.g., low mood, guilt, suicidal ideation) which were predicted both directly and indirectly by anxiety. The reverse depression-anxiety pathway did not support the observed moderated mediation relationship, nor did other dimensions of anger including frequency and severity feature in the prediction of affective depression symptoms. Taken together, this suggests that it is the social consequences of anger (e.g., problems getting along with others due to anger), rather than the emotion of anger itself, that contributes to affective depression symptoms in men with prostate cancer endorsing higher loneliness.

Despite associations with a range of negative psychosocial outcomes, anger has been a largely neglected emotion in mental health research (Eckhardt et al., 2004). Given research indicating anger's particular salience among men who adhere to Western masculine norms of strength, competitiveness, stoicism and invulnerability (Berke \& Zeichner, 2016), it deserves greater research attention in psycho-oncology, especially regarding prostate cancer-which is known to impact men's identity and sense of masculine self. Given the present findings highlight the relational aspects of men's experience, there are also implications for the partners of men with prostate cancer (Badger et al., 2011), both in terms of partner impacts and potential for reducing men's loneliness and anger. It is also important to note that iatrogenic effects of prostate cancer treatment are potential precipitants of men's anxiety, which may manifest in anger. For example, treatment induced sexual dysfunction, urinary incontinence and consequent relationship challenges amplifying changes to men's masculine identities may trigger states of anxiety, with anger-related social consequences in turn fuelling depression and loneliness. Accordingly, assessing for the presence and consequence of anger among men with prostate cancer (possibly using the DAR-5), and providing this population with appropriate avenues to process their affective state (e.g., gender specific and transformative interventions) is a potential pathway to boosting quality of life, social connectedness and functioning. Men with prostate cancer who have a tendency to suppress negative emotion also report a stronger association between symptoms of anger and major depression (Rice et al., 2020). If emotion suppression accentuates the angerdepression relationship, then approaches that lead to acceptance or reappraisal may offer more effective management strategies (Chambers et al., 2012).

Much of the rhetoric related to prostate cancer positions men's reluctance to engage in psychosocial oncology care as reflecting their alignment to masculine ideals of self-reliance and stoicism, and/or the lack of men-sensitive services. In highlighting anger as social interference, a gender paradox emerges, supported by stronger associations between anger and mental ill health among older men, relative to older women (Zebhauser et al., 2014). While anger has been normed and often legitimized as a masculine practice, it also increasingly draws critique and distance from others' for its negativity and threat. Therefore, de-stigmatizing anger in and of itself is 
key to therapeutically working with (rather than suppressing) emotion. One approach that may have particular relevance here is acceptance and commitment therapy that targets psychological flexibility, and has recently been shown to not only predict distress in men with prostate cancer, but also to moderate the effect of physical symptoms and masculine self-esteem on this distress (McAteer \& Gillanders, 2019). Additionally, there is scope to upskill allied health practitioners who work with men, where anger may be a key or presenting clinical concern. Such approaches could look to augment practice so that clinicians remain sensitized and responsive to anger in men with prostate cancer from a positon of curiosity (and where appropriate, empathy) rather than unease or avoidance (Seidler et al., 2021). Consistent with recent Delphi-based practitioner guidelines for prostate cancer survivorship (Dunn et al., 2020), skill development is likely to support personal agency related to self-awareness and ownership of health related needs. Techniques for adaptive anger management may result in a lower likelihood of men's interpersonal relationships being impacted as a consequence (Oliffe et al., 2021).

Interpretations from the present study are limited due to the use of cross-sectional convenience sampling comprising a relatively small sample who self-identified prostate cancer, with diagnoses not confirmed via medical record. Without healthy controls, we cannot be certain if these associations would also be observed in the general community, or are specific to men with prostate cancer. It is possible that stage/progression of illness may moderate effects, but unfortunately this data were not collected from participants. Considering the role of comorbidities and other health challenges in these associations will also be important for future research. While the study used well validated measures of anxiety and depression symptoms, the GAD-7 and PHQ-9 response scales assess for symptom frequency rather than severity, and different results may be observed in measures focussing on severity, or through diagnoses verified by clinical interview. Despite these limitations, the article has a number of strengths. Findings for anxiety and depression were not confounded by being on current treatment, receiving ADT or time since diagnosis, as there was no association for these parameters. Further, the present study included a holistic assessment of anger dimensions, with results providing insights into largely neglected areas of research with men experiencing prostate cancer and providing directions for future research to further explore and confirm.

In conclusion, findings indicate that anger-related social interference (as distinct from anger frequency, intensity, duration or antagonism) may be key to explaining the previously established anxiety-depression pathway for men with prostate cancer. However, this indirect relationship was conditional on loneliness, and was only observed for affective depression symptoms. Addressing the broader psychosocial needs of men experiencing prostate cancer may reduce the likelihood or severity of affective symptoms, and such approaches should consider anger and loneliness as potential therapeutic targets.

\section{Authors' contributions}

All authors have read and approved the final manuscript.

\section{Declaration of Conflicting Interests}

The author(s) declared no potential conflicts of interest with respect to the research, authorship, and/or publication of this article.

\section{Funding}

The author(s) disclosed receipt of the following financial support for the research, authorship, and/or publication of this article: This study was supported by the Canadian Institutes of Health Research (Grant 11R06913) and the open access article fee is made possible through John Oliffe's Tier 1 Canada Research Chair in Men's Health Promotion. Simon Rice was supported by a Career Development Fellowship from the National Health and Medical Research Council of Australia (GNT1158881), and the Dame Kate Campbell Fellowship from the Faculty of Medicine, Dentistry and Health Sciences at The University of Melbourne.

\section{ORCID iDs}

Zac E. Seidler (iD) https://orcid.org/0000-0002-6854-1554

Gabriela Montaner (iD https://orcid.org/0000-0002-8321-6688

John L. Oliffe (iD https://orcid.org/0000-0001-9029-4003

\section{Supplemental Material}

Supplemental material for this article is available online.

\section{References}

American Psychiatric Association. (2013). Diagnostic and statistical manual of mental disorders (DSM-5®). American Psychiatric Publications.

Badger, T. A., Segrin, C., Figueredo, A. J., Harrington, J., Sheppard, K., Passalacqua, S., \& Bishop, M. (2011). Psychosocial interventions to improve quality of life in prostate cancer survivors and their intimate or family partners. Quality of Life Research, 20(6), 833-844.

Baider, L., Ever-Hadani, P., Goldzweig, G., Wygoda, M. R., \& Peretz, T. (2003). Is perceived family support a relevant variable in psychological distress? A sample of prostate and breast cancer couples. Journal of Psychosomatic Research, 55(5), 453-460.

Berke, D. S., \& Zeichner, A. (2016). Man's heaviest burden: A review of contemporary paradigms and new directions for understanding and preventing masculine aggression. Social and Personality Psychology Compass, 10(2), 83-91. 
Beutel, M. E., Klein, E. M., Brähler, E., Reiner, I., Jünger, C., Michal, M., \& Lackner, K. J. (2017). Loneliness in the general population: prevalence, determinants and relations to mental health. BMC Psychiatry, 17(1), 97.

Brod, S., Rattazzi, L., Piras, G., \& D’Acquisto, F. (2014). 'As above, so below'examining the interplay between emotion and the immune system. Immunology, 143(3), 311-318.

Brunckhorst, O., Hashemi, S., Martin, A., George, G., Van Hemelrijck, M., Dasgupta, P., \& Ahmed, K. (2021). Depression, anxiety, and suicidality in patients with prostate cancer: A systematic review and meta-analysis of observational studies. Prostate Cancer and Prostatic Diseases, 24(2), 281-289.

Canadian Cancer Society. (2021). Survival statistics for prostate cancer. Retrieved from. https://www.cancer.ca/en/cancerinformation/cancer-type/prostate/prognosis-andsurvival/ survival-statistics/?region $=$ on.

Carter, N., Bryant-Lukosius, D., DiCenso, A., Blythe, J., \& Neville, A. J. (2011). The supportive care needs of men with advanced prostate cancer. Paper presented at the Oncology Nursing Forum.

Chambers, S. K., Foley, E., Galt, E., Ferguson, M., \& Clutton, S. (2012). Mindfulness groups for men with advanced prostate cancer: A pilot study to assess feasibility and effectiveness and the role of peer support. Supportive Care in Cancer, 20(6), 1183-1192.

Chambers, Hyde, M. K., Laurie, K., Legg, M., Frydenberg, M., Davis, I. D., . . Dunn, J. (2018). Experiences of Australian men diagnosed with advanced prostate cancer: A qualitative study. BMJ Open, 8(2).

Domènech-Abella, J., Mundó, J., Haro, J. M., \& Rubio-Valera, M. (2019). Anxiety, depression, loneliness and social network in the elderly: Longitudinal associations from The Irish Longitudinal Study on Ageing (TILDA). Journal of Affective Disorders, 246, 82-88.

Dunn, J., Green, A., Ralph, N., Newton, R. U., \& Kneebone, A. (2020). Prostate cancer survivorship essentials framework: guidelines for practitioners. Prostate, 1, 1-2020.

Eckhardt, C., Norlander, B., \& Deffenbacher, J. (2004). The assessment of anger and hostility: A critical review. Aggression and Violent Behavior, 9(1), 17-43.

Eid, M., Gollwitzer, M., \& Schmitt, M. (2017). Statistik und forschungsmethoden. Psychologie Verlagsunion.

Ervik, B., Nordøy, T., \& Asplund, K. (2010). Hit by wavesliving with local advanced or localized prostate cancer treated with endocrine therapy or under active surveillance. Cancer Nursing, 33(5), 382-389.

Fitch, M. I., Gray, R., Franssen, E., \& Johnson, B. (2000). Men's perspectives on the impact of prostate cancer: Implications for oncology nurses. Paper presented at the Oncology nursing forum.

Forbes, D., Alkemade, N., Hopcraft, D., Hawthorne, G., O'Halloran, P., Elhai, J. D., \& Bryant, R. (2014). Evaluation of the Dimensions of Anger Reactions-5 (DAR-5) Scale in combat veterans with posttraumatic stress disorder. Journal of Anxiety Disorders, 28(8), 830-835.

Frances, A. J., Galanter, M., \& Kleber, H. D. (2000). Diagnostic and Statistical Manual of Mental Disorders: DSM-IV-TR ${ }^{\circledR}$ : American Psychiatric Pub.
Galon, J., Pagès, F., Marincola, F. M., Thurin, M., Trinchieri, G., Fox, B. A., \& Ascierto, P. A. (2012). The immune score as a new possible approach for the classification of cancer. Journal of Translational Medicine, 10, 1.

George, L. K., Blazer, D. G., Hughes, D. C., \& Fowler, N. (1989). Social support and the outcome of major depression. The British Journal of Psychiatry, 154(4), 478-485.

Gerhart, J., Schmidt, E., Lillis, T., O’Mahony, S., Duberstein, P., \& Hoerger, M. (2017). Anger proneness and prognostic pessimism in men with prostate cancer. American Journal of Hospice and Palliative Medicine, 34(6), 497-504.

Hayes, A. F. (2017). Introduction to mediation, moderation, and conditional process analysis: A regression-based approach: Guilford publications.

Hinz, A., Mehnert, A., Kocalevent, R.-D., Brähler, E., Forkmann, T., Singer, S., \& Schulte, T. (2016). Assessment of depression severity with the PHQ-9 in cancer patients and in the general population. BMC Psychiatry, 16(1), 22.

Jacobson, N. C., \& Newman, M. G. (2017). Anxiety and depression as bidirectional risk factors for one another: A meta-analysis of longitudinal studies. Psychological Bulletin, 143(11), 1155.

Jaremka, L. M., Andridge, R. R., Fagundes, C. P., Alfano, C. M., Povoski, S. P., Lipari, A. M., \& Yee, L. D. (2014). Pain, depression, and fatigue: Loneliness as a longitudinal risk factor. Health Psychology, 33(9), 948.

King, A., Evans, M., Moore, T., Paterson, C., Sharp, D., Persad, R., \& Huntley, A. (2015). Prostate cancer and supportive care: A systematic review and qualitative synthesis of men's experiences and unmet needs. European Journal of Cancer Care, 24(5), 618-634.

Kroenke, K., Spitzer, R. L., \& Williams, J. B. (2001). The PHQ9: validity of a brief depression severity measure. Journal of General Internal Medicine, 16(9), 606-613.

Lehto, U.-S., Tenhola, H., Taari, K., \& Aromaa, A. (2017). Patients' perceptions of the negative effects following different prostate cancer treatments and the impact on psychological well-being: A nationwide survey. British Journal of Cancer, 116(7), 864-873.

McAteer, G., \& Gillanders, D. (2019). Investigating the role of psychological flexibility, masculine self-esteem and stoicism as predictors of psychological distress and quality of life in men living with prostate cancer. European Journal of Cancer Care, 28(4), e13097.

Novaco, R. W., Swanson, R. D., Gonzalez, O. I., Gahm, G. A., \& Reger, M. D. (2012). Anger and postcombat mental health: Validation of a brief anger measure with US Soldiers postdeployed from Iraq and Afghanistan. Psychological Assessment, 24(3), 661.

Oliffe, J. L., Kelly, M. T., Gonzalez Montaner, G., Seidler, Z. E., Maher, B., \& Rice, S. M. (2021). Men building better relationships: A scoping review. Health Promotion Journal of Australia. https://doi.org/10.1002/hpja.463

Oliffe, J. L., Ogrodniczuk, J., Bottorff, J. L., Hislop, T. G., \& Halpin, M. (2009). Connecting humor, health, and masculinities at prostate cancer support groups. Psycho-Oncology, 18(9), 916-926.

Oliffe, J. L., Rossnagel, E., Seidler, Z. E., Kealy, D., Ogrodniczuk, J. S., \& Rice, S. M. (2019). Men's Depression and Suicide. Current Psychiatry Reports, 21(10), 103. 
Overall, N. C., Hammond, M. D., McNulty, J. K., \& Finkel, E. J. (2016). When power shapes interpersonal behavior: Low relationship power predicts men's aggressive responses to low situational power. Journal of Personality and Social Psychology, 111(2), 195.

Penedo, F. J., Dahn, J. R., Kinsinger, D., Antoni, M. H., Molton, I., Gonzalez, J. S., \& Schneiderman, N. (2006). Anger suppression mediates the relationship between optimism and natural killer cell cytotoxicity in men treated for localized prostate cancer. Journal of Psychosomatic Research, 60(4), 423-427.

Resnick, M. J., \& Penson, D. F. (2012). Quality of life with advanced metastatic prostate cancer. Urologic Clinics, 39(4), 505-515.

Rice, S. M., Fallon, B. J., Aucote, H. M., \& Möller-Leimkühler, A. M. (2013). Development and preliminary validation of the male depression risk scale: Furthering the assessment of depression in men. Journal of Affective Disorders, 151(3), 950-958.

Rice, S. M., Kealy, D., Ogrodniczuk, J. S., Seidler, Z. E., Denehy, L., \& Oliffe, J. L. (2020). The cost of bottling it up: Emotion suppression as a mediator in the relationship between anger and depression among men with prostate cancer. Cancer Management and Research, 12, 1039.

Rice, S. M., Kealy, D., Oliffe, J. L., Seidler, Z. E., \& Ogrodniczuk, J. S. (2019). Affective-somatic symptoms of depression, suicide risk and exposure to childhood maltreatment: Data from emerging adults to older-age males. International Journal of Mental Health and Addiction, 17(6), 1301-1311.

Rice, S. M., Oliffe, J. L., Kelly, M. T., Cormie, P., Chambers, S., Ogrodniczuk, J. S., \& Kealy, D. (2018). Depression and prostate cancer: Examining comorbidity and male-specific symptoms. American Journal of Men's Health, 12(6), 1864-1872.

Russell, D. W. (1996). UCLA Loneliness Scale (Version 3): Reliability, validity, and factor structure. Journal of Personality Assessment, 66(1), 20-40.

Santini, Z. I., Fiori, K. L., Feeney, J., Tyrovolas, S., Haro, J. M., \& Koyanagi, A. (2016). Social relationships, loneliness, and mental health among older men and women in Ireland: A prospective community-based study. Journal of Affective Disorders, 204, 59-69.

Seidler, Z., Wilson, M., Trail, K., Rice, S., Kealy, D., Ogrodniczuk, J., \& Oliffe, J. L. (2021). Challenges working with men: Australian therapists' perspectives. Submitted.

Sharp, L., O’Leary, E., Kinnear, H., Gavin, A., \& Drummond, F. J. (2016). Cancer-related symptoms predict psychological wellbeing among prostate cancer survivors: Results from the PiCTure study. Psycho-Oncology, 25(3), 282-291.
Sharpley, C. F., Bitsika, V., \& Christie, D. R. (2007). Causal'mapping' of depression and anxiety among prostate cancer patients: A preliminary interview study. Journal of Men's Health and Gender, 4(4), 402-408.

Sharpley, C. F., \& Christie, D. R. (2007). An analysis of the psychometric profile and frequency of anxiety and depression in Australian men with prostate cancer. Psycho-Oncology, 16(7), 660-667.

Sharpley, C. F., Christie, D. R., \& Bitsika, V. (2010). Variability in anxiety and depression over time following diagnosis in patients with prostate cancer. Journal of Psychosocial Oncology, 28(6), 644-665.

Spitzer, R. L., Kroenke, K., Williams, J. B., \& Löwe, B. (2006). A brief measure for assessing generalized anxiety disorder: The GAD-7. Archives of Internal Medicine, 166(10), 1092-1097.

Steptoe, A., Shankar, A., Demakakos, P., \& Wardle, J. (2013). Social isolation, loneliness, and all-cause mortality in older men and women. Proceedings of the National Academy of Sciences, 110(15), 5797-5801.

Tymoszuk, U., Perkins, R., Fancourt, D., \& Williamon, A. (2019). Cross-sectional and longitudinal associations between receptive arts engagement and loneliness among older adults. Social Psychiatry and Psychiatric Epidemiology, 55, 891900.

Vasiliadis, H.-M., Chudzinski, V., Gontijo-Guerra, S., \& Préville, M. (2015). Screening instruments for a population of older adults: The 10-item Kessler Psychological Distress Scale (K10) and the 7-item Generalized Anxiety Disorder Scale (GAD-7). Psychiatry Research, 228(1), 89-94.

Von Elm, E., Altman, D. G., Egger, M., Pocock, S. J., Gøtzsche, P. C., \& Vandenbroucke, J. P. (2007). The strengthening the reporting of observational studies in epidemiology (STROBE) statement: guidelines for reporting observational studies. Annals of Internal Medicine, 147(8), 573-577.

Watts, S., Leydon, G., Birch, B., Prescott, P., Lai, L., Eardley, S., \& Lewith, G. (2014). Depression and anxiety in prostate cancer: a systematic review and meta-analysis of prevalence rates. BMJ Open, 4(3), e003901.

White, V. M., English, D. R., Coates, H., Lagerlund, M., Borland, R., \& Giles, G. G. (2007). Is cancer risk associated with anger control and negative affect? Findings from a prospective cohort study. Psychosomatic Medicine, 69(7), 667-674.

Zebhauser, A., Hofmann-Xu, L., Baumert, J., Häfner, S., Lacruz, M., Emeny, R., \& Peters, A. (2014). How much does it hurt to be lonely? Mental and physical differences between older men and women in the KORA-Age Study. International Journal of Geriatric Psychiatry, 29(3), 245-252. 


\section{University Library}

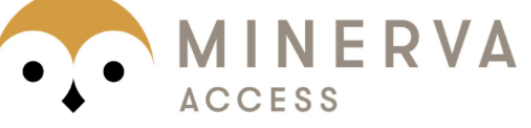

A gateway to Melbourne's research publications

Minerva Access is the Institutional Repository of The University of Melbourne

Author/s:

Rice, SM;Kealy, D;Ogrodniczuk, JS;Seidler, ZE;Montaner, G;Chambers, S;Oliffe, JL

Title:

The Anxiety Depression Pathway Among Men Following a Prostate Cancer Diagnosis: Cross-Sectional Interactions Between Anger Responses and Loneliness

Date:

2021-05-01

Citation:

Rice, S. M., Kealy, D., Ogrodniczuk, J. S., Seidler, Z. E., Montaner, G., Chambers, S. \& Oliffe, J. L. (2021). The Anxiety Depression Pathway Among Men Following a Prostate Cancer Diagnosis: Cross-Sectional Interactions Between Anger Responses and Loneliness. AMERICAN JOURNAL OF MENS HEALTH, 15 (3), https:// doi.org/10.1177/15579883211023699.

Persistent Link:

http://hdl.handle.net/11343/281184

License:

CC BY-NC 